\section{Characteristics of non-vitreoretinal ocular injury in child maltreatment: a systematic review}

T Betts ${ }^{1}$, S Ahmed ${ }^{2}$, S Maguire ${ }^{3}$ and P Watts ${ }^{4}$

highlight further specific characteristics of non-vitreoretinal ocular injury indicative of child abuse.

Eye (2017) 31, 1146-1154; doi:10.1038/eye.2017.25; published online 24 March 2017

\section{Introduction}

Child maltreatment includes all forms of child abuse and neglect, in addition to fabricated or induced illness. The latter is often difficult to detect and may thus go unrecognised.

The National Society for the Prevention of Cruelty to Children survey data reported that $6.9 \%$ young people aged 11-17 had experienced abuse or neglect at the hands of their parents or guardians. In addition, $\sim 70$ children a year die of maltreatment in the UK, with the majority of these being aged $<5$ years. ${ }^{1}$

Ocular signs and symptoms in child abuse may manifest as a result of direct or indirect injury or sexually transmitted infections.

Thus far studies on the ocular signs have focused on vitreoretinal injury associated with abusive head trauma. The retinal features of this injury have been well described, in recent systematic reviews. ${ }^{2,3}$ However there is a paucity of data on non-vitreoretinal ocular abusive injury.

Accidental ocular injury among children requiring hospitalisation in Scotland during 1996 had an incidence of 8.14 per $100000 .{ }^{4}$ In Maryland, USA, the incidence during 1982 was 15.2 per $100000 .^{5}$ The majority of these injuries were non-penetrating injuries with resultant hyphaemas. None of these epidemiological studies have reported child maltreatment as a cause of ocular injury, thus making true estimates of its prevalence difficult to ascertain.

Ocular injuries can be sustained from a wide range of mechanisms including blunt trauma, 
penetrating injuries and burns. Non-vitreoretinal ophthalmic injury in child maltreatment varies and the diagnosis may prove challenging. Paediatricians, emergency physicians or ophthalmologists may be called upon to assess a child presenting with such injuries. The challenge is to identify which non-vitreoretinal ocular injury in children may be abusive.

To date there have been no published systematic reviews of non-vitreoretinal ocular injuries in child maltreatment. The safeguarding of children remains the responsibility of all clinicians, with mandatory reporting of suspected maltreatment in many countries worldwide. In the UK all clinician's have a duty of care to report suspected injury due to maltreatment. This systematic review was undertaken to identify the spectrum of nonretinal ocular abusive injury.

\section{Materials and methods}

The inclusion criteria consisted of studies of children aged 0-18 years experiencing non-vitreoretinal ocular injury due to physical abuse or fabricated or induced illness, with adequate confirmation of the cause of injury and details of the injuries sustained (see quality standards below).

An all language literature search was conducted across the databases MEDLINE, PsychINFO, EMBASE, AMED, Web of Science, and CINAHL for the period 1950 to January 2015. All observational study types were included after a scoping search, as it was evident that high-quality comparative studies were lacking. The search strategy was developed in Ovid Medline, and adapted for the remaining databases. The strategy used four sets of key words and MESH headings relating to childhood, child maltreatment, injuries, and ocular terminology (Supplementary Appendix 1).

Identified articles were transferred to Endnote X7, at which point duplicate studies were removed manually. The retrieved articles were screened using title and abstract to remove irrelevant articles by authors SA and SM. Full texts of the remaining articles were then retrieved using a variety of sources. Around 28 articles were unobtainable. All full texts were scanned, and studies matching the inclusion criteria underwent full review by two reviewers, with double data extraction. All articles were appraised using a standardised critical appraisal form (Supplementary Appendix 2) and information was transferred to Microsoft Excel 2013. Inclusion disagreement by the primary investigators following critical appraisal was resolved by third review. See PRISMA flow diagram for studies retrieved (Figure 1). Snowballing of included article references was performed.

\section{Quality standards}

It was imperative that studies included had a confirmed abusive aetiology. Therefore we adopted a ranking of maltreatment as per previous published standards. ${ }^{2}$ This review included studies of rank 1 or 2 to minimise the risk of 'circularity' by not relying solely on clinical features (Table 1). Any study that included cases of 'suspected' abuse or that relied solely on physical findings without a multidisciplinary assessment, were therefore excluded.

The ranking of ocular examination was applied to ensure that the included articles met the expected standard of clinical examination. We excluded all publications where the children had not been examined by an ophthalmologist (rank 1), or a healthcare worker that performed regular ocular examinations (rank 2) (Table 1). The misdiagnosis of ocular complaints and missed clinical findings by the non-ophthalmologists is well reported. 6

Articles were also excluded if the additional criteria outlined in Table 2 were met.

Authors Derider et $a l^{7}$ were contacted to clarify quality standards with regards to confirmation of child abuse and whether ocular examination standards were met, which led to exclusion of a single case (Case 6). Information was extracted from each study on the form of maltreatment and ranking, ocular examination, history, and clinical findings.

\section{Narrative synthesis}

The primary outcome measure of the systematic review was to describe characteristic patterns of ocular injury in child maltreatment, aiming to alert the healthcare worker of any particular characteristic clinical features on ocular clinical examination findings that may be indicative of maltreatment.

A meta-analysis of extracted data was not possible due to the lack of comparative data in the literature. Extracted data were therefore tabulated and combined for narrative synthesis.

\section{Results}

Of the 49 articles reviewed, five met the inclusion criteria. There were three case series and two case reports. ${ }^{7-11}$ The principal reason for exclusion was the absence of a nonvitreoretinal ocular injury.

We have summarised the results according to three categories: 
PRISMA 2009 Flow Diagram
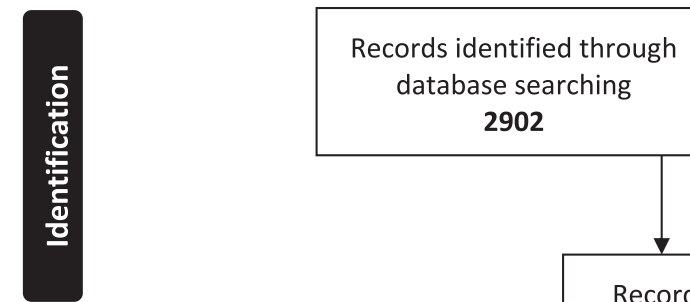

2902

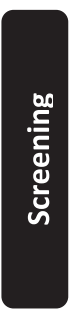

Records after duplicates removed

1492
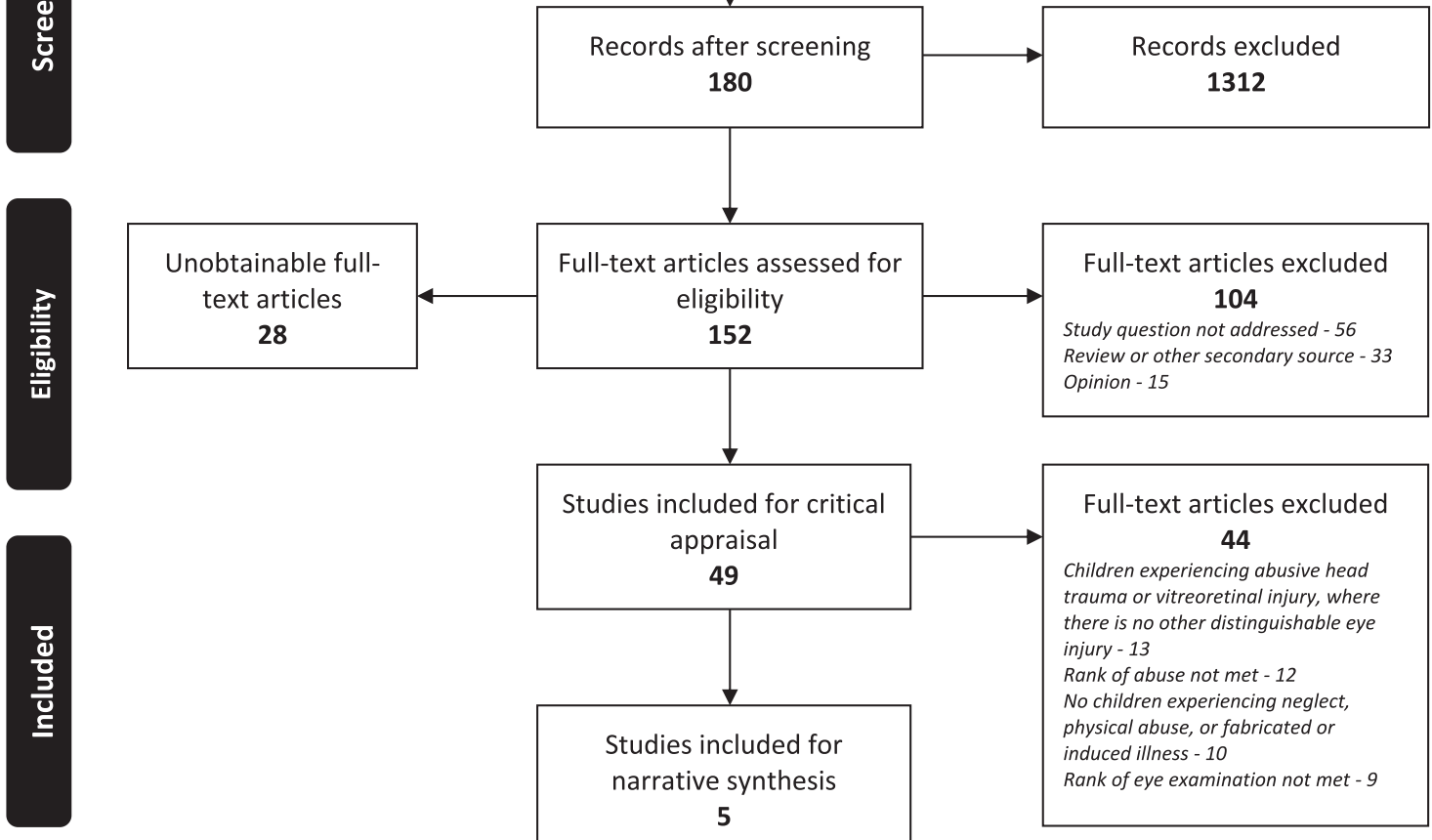

Full-text articles excluded

dditional records identified through other sources 0

0

Figure 1 Preferred Reporting Items for Systematic Reviews and Meta-Analyses (PRISMA) flow diagram of review process in a systematic review of the characteristics of eye injuries in child maltreatment.

Data set 1: Ocular injury as a consequence of physical abuse.

Data set 2: Ocular injury as a result of fabricated or induced illness (FII).

Data set 3: Ocular injury as a result of corporal punishment.

The total data set includes 26 children, 14 male, and 12 female. The mean age across the included articles was 36.0 months, range 1.0-168.0 months. Excluding data set three the mean age of the children is 13.9 months, range 1.0-68.0 months. Confirmation of maltreatment was ranked one for 13 children, and 13 as rank 2 (Table 1). All children had a rank 1 ophthalmic examination (Table 1).
Ocular injury as a consequence of physical abuse (Data set 1)

This comprises three articles by DeRidder et $a l^{7}$ Spitzer et $a l^{8}$ and Skarbek-Borowska et $a l^{9}$ publishing on a total of 18 cases of ocular injury in child abuse. DeRidder and Spitzer's cases are limited to subconjunctival haemorrhages as the presenting sign and SkarbekBorowska presents globe ruptures with associated anterior segment signs.

DeRidder et $a l^{7}$ present 13 children aged 1-68 month (mean age 17.3 month, median 7 month) eight boys and five girls. Five children had bilateral subconjunctival haemorrhages and eight were unilateral. One child had periorbital oedema, one a polar cataract, one optic nerve 
Table 1 The rank of confirmation of abuse and quality of eye examination in a systematic review of the characteristics of ocular injuries in child maltreatment

\begin{tabular}{|c|c|c|}
\hline Rank classification & Rank of abuse & Rank of ophthalmic examination \\
\hline 1 & $\begin{array}{l}\text { Abuse confirmed at case conference, family, civil or } \\
\text { criminal court proceedings, admitted by perpetrator or } \\
\text { independently witnessed }\end{array}$ & An ophthalmologist \\
\hline 2 & $\begin{array}{l}\text { Abuse confirmed by stated/ referenced criteria including } \\
\text { multi-disciplinary assessment }\end{array}$ & $\begin{array}{l}\text { A healthcare professional who regularly examines the } \\
\text { eye. Details must be provided. (eg optometrist, } \\
\text { emergency physician, primary care physician with special } \\
\text { interest in eyes) }\end{array}$ \\
\hline 3 & Abuse defined by stated criteria & $\begin{array}{l}\text { Other healthcare professionals who do not regularly } \\
\text { examine the eye }\end{array}$ \\
\hline 4 & Abuse stated but no supporting detail given & No detail given \\
\hline 5 & Suspected abuse & \\
\hline
\end{tabular}

Table 2 Article exclusion criteria in a systematic review of the characteristics of ocular injuries in child maltreatment

Exclusion criteria

Review article

Expert opinion

Study addresses adults only: (population over 18 years) or mixed adult and child population where child related data cannot be extracted

Eye injury exclusively caused by sexual abuse, where abuse type is not specified, or mixed cases of physical/sexual abuse/neglect are present and cannot be separated

No children experiencing neglect, physical abuse, or fabricated or induced illness

Children experiencing abusive head trauma or vitreoretinal injury, where there is no distinguishable eye injury

Eye injury caused by substance abuse during pregnancy

Study describes prematurity or birth related eye trauma

Patient(s) deemed to have organic disease causing the eye injury pattern

Injuries of war or disaster

No eye injury present

elevation. One of the cases had presented to the emergency department with a corneal abrasion, the cause of which is not discussed. Ten cases had associated bruising; this included facial, abdominal and limb bruising and one of whom had a burn to the foot. Skeletal survey was recorded in all 13 children, six of whom had fractures (summarised in Table 3). Neuro-radiological investigations 'were performed at the discretion of the attending physician', and it is unclear how many underwent neuroimaging. The reported positive findings identified a subarachnoid haemorrhage; one hypoxic ischaemic encephalopathy and two children had facial soft tissue swellings/haematoma. One child was found to have a liver laceration on abdominal computed tomography. Abnormal coagulation laboratory findings were reported in one case.

The mechanism of injury is stated in four cases, two children having been struck, one pushed down stairs, and one 'caught by arm'.

Spitzer $\mathrm{et}^{\mathrm{al}} \mathrm{l}^{8}$ present three children aged 5 weeks to 5 months (mean age 3.7 months) two boys and one girl. All children had bilateral subconjunctival haemorrhages and otherwise normal ocular examinations. Two infants presented initially to the heath care services with bilateral subconjunctival haemorrhages of no attributable cause. In one case child maltreatment was suspected, but investigations including skeletal survey and neuroimaging were found to be normal. Having been discharged, they both represented within one month, one child with rib fractures and one with long bone fractures.

At the time of reporting, all three children underwent a skeletal survey and one child had neuroimaging, which was unremarkable.

These injuries were speculated in one case to be the result of chest compression and unknown in the other two cases.

Skarbek-Borowska et $a l^{9}$ present two cases where globe rupture was the presenting feature of maltreatment. The children (both girls) were aged 9 and 14 months (mean and median 11.5 months). Both cases presented with extensive chemosis, subconjunctival haemorrhage, and total hyphaema. Globe rupture was confirmed during examination under anaesthesia and subsequent 


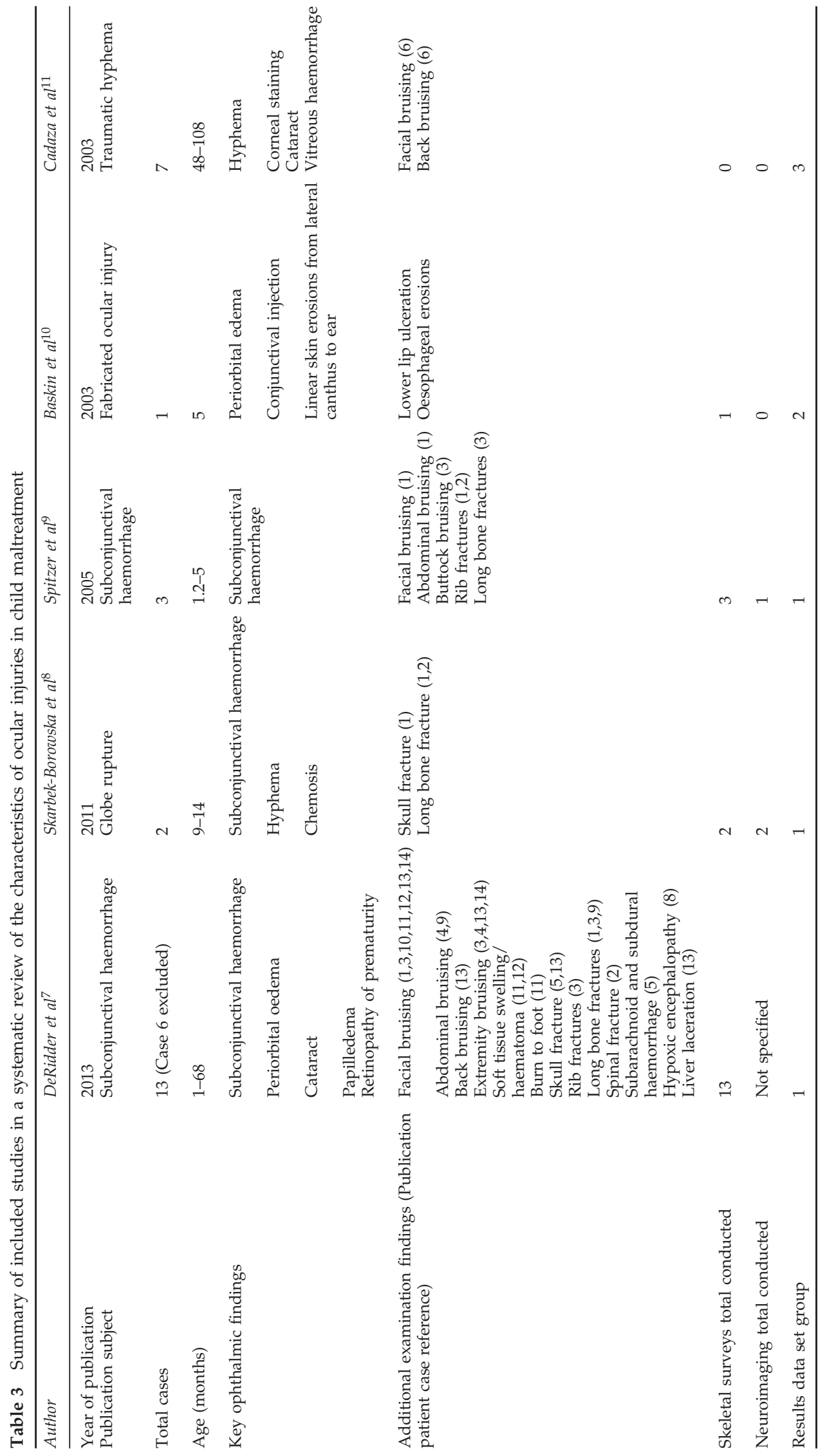


neuroimaging and skeletal survey findings are summarised in Table 3. In one of these cases it is noted that the child had been seen a week previously at a local clinic with the ocular complaint and diagnosed with conjunctivitis.

The globe rupture was reported to have occurred as a result of direct pressure from a digit in one case and is unknown in the other.

In summary, all of the cases $(18 / 18)$ in this data set were found to have subconjunctival haemorrhage of one or both eyes as a presenting sign of physical maltreatment. It is notable that $22 \%(4 / 18)$ of cases had been seen in the preceding weeks with an ocular complaint and discharged, without maltreatment being recognised.

\section{Ocular injuries as a consequence of Fabricated or Induced Illness (Data set 2)}

Data set 2 comprises of a single article in which a fabricated illness of the eye was the presenting feature in a case of child maltreatment. Baskin et al ${ }^{10}$ detail a 5-monthold female infant, who presented to a major hospital on three consecutive days with periorbital erythema and oedema, and was diagnosed initially with viral conjunctivitis and subsequently with otitis media. The child re-presented five days later with worsening eyelid signs, conjunctival injection and punctate corneal erosions. The presence of lip ulceration prompted admission for possible Steven-Johnson syndrome. The child's health improved on topical steroids and systemic supportive therapy and she was discharged well at three weeks. The following day the child was re-admitted with marked lid erythema and oedema and 'unusual linear cutaneous erosions' extending from the lateral canthus of the left eye to the left ear and a similar lesion at the right ear. An epithelial defect with underlying stromal haze was demonstrated on slit lamp examination; otherwise the ophthalmic examination was reported as normal. Investigation from other subspecialties found upper gastroenterological findings in keeping with the ingestion of a caustic agent.

The child's mother later admitted to inflicting the injuries with an undisclosed agent.

In summary this child underwent repeated examination, and on the fifth presentation a diagnosis of maltreatment was made following the identification of uncharacteristic ocular findings.

\section{Ocular injuries as a consequence of corporal punishment (Data set 3)}

Calzada et al $^{11}$ present a case series of children who developed traumatic hyphaema following corporal punishment with a belt. A total of seven children are included, five boys and two girls with an age range of 4.0-14.0 years (mean and median 8 years). On examination at presentation visual acuity ranged from $20 / 15$ to light perception (three cases, $43 \%$, counting fingers or worse) and hyphaema ranged from 5 to $85 \%$ (mean 24\%). In addition corneal bloodstaining was found in two cases, one with concurrent vitreous haemorrhage. A traumatic cataract was identified in another case and retinal commotio, angle recession, and traumatic mydriasis in one other. Co-existent injuries identified on extraocular examination included face and back ecchymoses in one case. Neither skeletal survey nor neuroimaging were conducted in any cases.

Conservative management was employed in all but one case, which underwent vitrectomy. A final visual acuity at time of reporting was $20 / 60$ or worse in $43 \%$ (3/7) of the cases. All children had been struck with a belt.

In summary these children are significantly older than those in Data sets 1 and 2. All had hyphaema following corporal punishment with a belt and there were frequently associated ocular and extra-ocular clinical findings. A large proportion of the children were left with significantly impaired vision. No child underwent imaging for occult skeletal or neurological injury.

\section{Discussion}

This comprehensive systematic review applied strict inclusion standards for both the ophthalmic examination and confidence in maltreatment diagnosis, which reflects the rigorous standards expected today both clinically and legally. Disappointingly however, the lack of comparative literature addressing this topic precluded a meta-analysis, and thus we have conducted a narrative synthesis on all data published of non-retinal ocular injury in cases of confirmed child maltreatment. The included cases describe a wide variety of ocular, facial and skeletal injuries occurring as a consequence of child maltreatment. It is of particular interest, however that all the children that had suffered physical abuse with ocular injury had subconjunctival haemorrhages. The subconjunctival haemorrhages in 10 cases were unilateral and in eight cases bilateral. Unfortunately there was no standardised reporting of this finding and so no further descriptive detail can be ascertained. The extensive list of aetiologies presenting with subconjunctival haemorrhages in childhood is well reported. Causes include infectious, haematological and neoplastic disease, Valsalva manoeuvre, vomiting, and accidental trauma. ${ }^{712}$ In the absence of these aetiologies non-accidental injury must be considered.

It was also observed that preceding the diagnosis of non-accidental injury, a notable proportion had been seen with an ocular complaint and maltreatment missed. This 
is in line with published estimates of almost a third of children presenting with physical abuse failing to be identified at their initial presentation. ${ }^{13}$ The review raises the possibility of subconjunctival haemorrhages as a 'sentinel injury' in child maltreatment, as has previously been described for bruising or a torn labial frenum. ${ }^{14-16}$ The identification of 'sentinel injuries' in children who later suffer severe physical maltreatment has become increasingly appreciated in recent years. ${ }^{15}$ These are minor injuries such as bruising or intraoral injury in precruising children, which are clearly visible to caregiver or medical practitioner and poorly explained or unexpected. In 2013 Sheets et al published a retrospective case-control study evaluating the incidence of such injuries in abused and non-abused children. ${ }^{16}$ They found $27.5 \%$ of definitely abused children had a previous sentinel injury compare to no children in the non-abused group. The limited cases that this systematic review has provided appears to support such a pattern, with $26.3 \%$ of children seen in the preceding weeks with an ocular complaint and discharged, without maltreatment being recognised (Data sets 1 and 2 only).

The systematic review demonstrated that FII and corporal punishment may present with an ocular complaint. Children presenting with ocular injuries from maltreatment had a mean age of 13.9 months, median 6 months and range 1-68. While those who suffered corporal punishment resulting in ocular injury, were considerably older (mean and median 96 months).

The corporal punishment of children is currently prohibited in 44 countries, having first been banned in Sweden in 1979. There is no current ban on corporal punishment in the UK, where 'reasonable chastisement' by parents is allowed. However, assaulting your child with a belt such that significant ocular injuries occurs would appear to exceed this threshold.

All cases of maltreatment in data sets 1 and 2 underwent skeletal survey, but we could confirm in only two cases of children $<1$ year that neuroimaging had taken place. The current recommendation is that all children under two years with suspected maltreatment undergo a skeletal survery. ${ }^{17}$

The current indications for neuroimaging in suspected physical maltreatment includes all children under the age of one and those with encephalopathic features or focal neurological signs or haemorrhagic retinopathy. ${ }^{17}$ In the US, guidance for the radiological investigation of children suspected of suffering abusive injuries is less precise with 'appropriateness' guidelines produced by the American College of Radiology. ${ }^{18}$

Among those who had suffered corporal punishment, none underwent any form of further investigations, thus the prevalence of occult injury cannot be determined.
The mean age at presentation from the included data of maltreated children (Data set 1 and 2) was 13.9 months, while a US study from 1997-2006 found incidence of paediatric ocular injury from consumer products (including toys, sports, and so on) to be at its lowest in children under 2 years. ${ }^{19}$ This would suggest that ocular injury in this age group is at the least unusual, and warrants careful assessment.

There is a notable deficiency in epidemiological data of paediatric ocular trauma. Estimated incidence ranges from 8.9-15.2/100 000 for paediatric ocular trauma requiring hospital treatment. ${ }^{20}$ The most extensive studies focus on hospitalised children and therefore excludes up to $95 \%$ of ocular trauma that does not require admission. ${ }^{20}$ The majority of paediatric ocular injury will be seen in the emergency department making identification of potential abusive ocular injuries a priority.

The absence of substantial comparative data on accidental paediatric ocular trauma was apparent and it is encouraging to find that this work is currently being undertaken by the British Ophthalmological Surveillance Unit. To date the most extensive review article was published by Abott and Shah in 2012 with the common causes of paediatric ocular injury being from projectiles, sports-related, airbags and so on. Both penetrating and blunt trauma were demonstrated, with a male preponderance and ocular trauma was least frequent in those under the age of two. ${ }^{20}$ This is notable, as the most commonly reported abusive ocular injuries occurred in those aged $<2$ years.

Several commendable studies could not be included in this review having failed to meet the strict inclusion criteria. Unfortunately early publications were found to have inadequate confirmation of maltreatment that is expected today. ${ }^{21}$ Adopting standardised documentation of the ophthalmic examination would improve reporting and increase the potential for meta-analysis of abusive paediatric non-vitreoretinal ocular injuries in the future. ${ }^{2,22}$ It is to be hoped that this will become the norm in the UK at least following the publication of a standardised proforma for the documentation of all ocular examinations in cases of suspected maltreatment. ${ }^{22}$

In contrast to retinal haemorrhages, paediatric nonvitreoretinal ocular injuries are often readily identifiable. In spite of this, to reduce the incidence of under-reporting and missed inflicted injury, the involvement of an ophthalmologist should be sought in paediatric ocular trauma where doubt exists with regards its causation.

Of interest only three cases $(11.5 \%)$ from the included publications were found to have concurrent vitreoretinal pathology. Calzada et al ${ }^{11}$ describe a vitreous haemorrhage in a nine year old boy and retinal commotio in a fourteen year old female. Additionally DeRidder et $a l^{7}$ describe a 38 month old male with an 'elevated optic nerve'. 


\section{Summary}

The purpose of this systematic review was to identify the spectrum of non-vitreoretinal ocular abusive injuries and potentially highlight any particular charateristic injures, in contrast to accidental injuries. The narrative synthesis of available literature highlights that abusive ocular injuries were seen in children ranging in age from one month to fourteen years (for those experiencing severe corporal punishment) and the spectrum of injuries included periorbital oedema, chemosis, subconjunctival haemorrhage, corneal epithelial loss, hyphaema, cataract, and globe rupture. As clinicians this should heighten our awareness of the possibility of abusive ocular injuries, and we must ensure that appropriate enquiries are instigated where warranted.

While we may have identified subconjunctival haemorrhages as a potential sentinel injury of maltreatment, and an exploration of possible causes should include consideration of maltreatment, this conclusion is limited by the lack of large-scale studies of children with subconjunctival haemorrhages from all causes.

Standardisation of the ophthalmic examination and definitive confirmation of maltreatment are clearly essential standards for future work to enhance clinical practice, assist research into the field of ocular injury, and ultimately enhance prompt recognition of child maltreatment and appropriate referral for safeguarding interventions.

\section{Summary}

\section{What was known before}

- Ophthalmic clinicians encounter a wide variety of nonvitreoretinal ocular injuries. Vitreoretinal injury due to child maltreatment has been well described.

What this study adds

- Confirmation of the wide spectrum of non-vitreoretinal ocular injuries seen in child abuse or fabricated or induced illness, particularly among young children.

- $75 \%$ of included cases occurred in children $<4$ years of age.

- Unexplained subconjunctival haemorrhages may be a potential sentinel injury of maltreatment and warrant careful evaluation.

\section{Conflict of interest}

The authors declare no conflict of interest.

\section{Acknowledgements}

Dr Catherine DeRidder for her clarification of patient data. Funding from National Society for Prevention of Cruelty to Children (NSPCC), grant number 1755. National Society for the Prevention of Cruelty to Children
(NSPCC) funding, grant application number 1755. No equipment or drugs, no federal funding.

\section{References}

1 Jütte $\mathrm{S}$, Bentley $\mathrm{H}$, Tallis D, Mayes J, Jetha $\mathrm{N}$, $\mathrm{O}^{\prime}$ Hagan $\mathrm{O}$ et al. How safe are our children? The Most Comprehensive Overview of Child Protection in the UK. NSPCC: London, UK, 2015.

2 Maguire SA, Watts PO, Shaw AD, Holden S, Taylor RH, Watkins WJ et al. Retinal haemorrhages and related findings in abusive and non-abusive head trauma: a systematic review. Eye (Lond) 2013; 27: 28-36.

3 Bhardwaj G, Chowdhury V, Jacobs MB, Moran KT, Martin FJ, Coroneo MT. A systematic review of the diagnostic accuracy of ocular signs in pediatric abusive head trauma. Ophthalmology 2010; 117: 983-992.

4 Desai P, MacEwen CJ, Baines P, Minassian DC. Incidence of cases of ocular trauma admitted to hospital and incidence of blinding outcome. Br J Ophthalmol. 1996; 80: 592-596.

5 Strahlman E, Elman M, Daub E, Baker S. Causes of pediatric ocular injuries: a population-based study. Arch Ophthalmol 1990; 108: 603-606.

6 Morad Y, Kim YM, Mian M, Huyer D, Capra L, Levin AV. Nonophthalmologist accuracy in diagnosing retinal hemorrhages in the shaken baby syndrome. J Pediatr 2003; 142: 431-434.

7 DeRidder CA, Berkowitz CD, Hicks RA, Laskey AL. Subconjunctival hemorrhages in infants and children: a sign of nonaccidental trauma. Pediatr Emerg Care 2013; 29: 222-226.

8 Spitzer SG, Luorno J, Noël LP. Isolated subconjunctival hemorrhages in nonaccidental trauma. J AAPOS 2005; 9: $53-56$.

9 Skarbek-Borowska SE, Campbell KT. Globe rupture and nonaccidental trauma: two case reports. Pediatr Emerg Care 2011; 27: 544-546.

10 Baskin DE, Stein F, Coats DK, Paysse EA. Recurrent conjunctivitis as a presentation of munchausen syndrome by proxy. Ophthalmology 2003; 110: 1582-1584.

11 Calzada JI, Kerr NC. Traumatic hyphemas in children secondary to corporal punishment with a belt. Am J Ophthalmol 2003; 135: 719-720.

12 Tarlan B, Kiratli H. Subconjunctival hemorrhage: risk factors and potential indicators. Clin Ophthalmol 2013; 7: 1163-1170.

13 Thorpe EL, Zuckerbraun NS, Wolford JE, Berger RP. Missed opportunities to diagnose child physical abuse. Pediatr Emerg Care 2014; 30: 771-776.

14 Jenny C, Hymel KP, Ritzen A, Reinert SE, Hay TC. Analysis of missed cases of abusive head trauma. JAMA 1999; 281: 621-626.

15 Greiner MV, Berger RP, Thackeray JD, Lindberg DM. Examining Siblings to Recognize Abuse (ExSTRA) Investigators. Dedicated retinal examination in children evaluated for physical abuse without radiographically identified traumatic brain injury. J Pediatr 2013; 163: 527-531.

16 Petska HW, Sheets LK. Sentinel injuries: subtle findings of physical abuse. Pediatr Clin North Am 2014; 61: 923-935.

17 Standards for Radiological Investigations of Suspected Non-accidental Injury - March 2008. https:/ /www.rcr.ac. uk/sites/default/files/publication/RCPCH_RCR_final_0. pdf. Accessed 15/02/2016. 
18 American College of Radiology Appropriateness Criteria 2012. https:/ /acsearch.acr.org/docs/69443/Narrative/. Accessed 15/02/2016

19 Moren Cross J, Griffin R, Owsley C, McGwin G Pediatric ocular injuries related to consumer products in the United States. J AAPOS 2008; 12: 626-628.

20 Abbott J, Shah P. The epidemiology and etiology of pediatric ocular trauma. Surv Ophthalmol 2013; 58: 476-485.
21 Taylor D, Bentovim A. Recurrent nonaccidentally inflicted chemical eye injuries to siblings. J Pediatr Ophthalmol 1976; 13(4): 238-244.

22 Abusive head trauma and the eye. Royal College of Ophthalmologists 2013. https: / / www.rcophth.ac.uk/wpcontent/uploads/2014/12/2013-SCI-292-ABUSIVE-HEADTRAUMA-AND-THE-EYE-FINAL-at-June-2013.pdf. Accessed 15/02/2016.

Supplementary Information accompanies this paper on Eye website (http://www.nature.com/eye) 